\title{
Operation of an ADR using helium exchange gas as a substitute for a failed heat switch
}

\author{
P. Shirron ${ }^{\mathrm{a}, *}$, M. DiPirro ${ }^{\mathrm{a}}$, M. Kimball ${ }^{\mathrm{a}}$, G. Sneiderman ${ }^{\mathrm{a}}$, F.S. Porter ${ }^{\mathrm{a}}$, C. Kilbourne ${ }^{\mathrm{a}}$, R. Kelley ${ }^{\mathrm{a}}$, \\ R. Fujimoto ${ }^{\text {b }}$, S. Yoshida ${ }^{c}$, Y. Takei $^{\mathrm{d}}$, K. Mitsuda ${ }^{\mathrm{d}}$

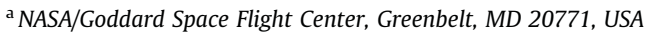 \\ ${ }^{\mathrm{b}}$ Institute of Science and Engineering, Kanazawa University, Ishikawa 920-1192, Japan

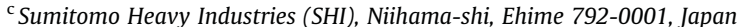 \\ ${ }^{\mathrm{d}}$ Institute of Space and Astronautical Science (ISAS), Japan Aerospace Exploration Agency (JAXA), Sagamihara, Kanagawa 252-5210, Japan
}

\section{A R T I C L E I N F O}

\section{Article history:}

Available online 26 April 2014

\section{Keywords:}

Adiabatic demagnetization

ADR

Space cryogenics

Exchange gas

\begin{abstract}
A B S T R A C T
The Soft X-ray Spectrometer (SXS) is one of four instruments on the Japanese Astro-H mission, which is currently planned for launch in late 2015. The SXS will perform imaging spectroscopy in the soft X-ray band (0.3-12 keV) using a $6 \times 6$ pixel array of microcalorimeters cooled to $50 \mathrm{mK}$. The detectors are cooled by a 3-stage adiabatic demagnetization refrigerator (ADR) that rejects heat to either a superfluid helium tank (at $1.2 \mathrm{~K}$ ) or to a $4.5 \mathrm{~K}$ Joule-Thomson (JT) cryocooler. Four gas-gap heat switches are used in the assembly to manage heat flow between the ADR stages and the heat sinks. The engineering model (EM) ADR was assembled and performance tested at NASA/GSFC in November 2011, and subsequently installed in the EM dewar at Sumitomo Heavy Industries, Japan. During the first cooldown in July 2012, a failure of the heat switch that linked the two colder stages of the ADR to the helium tank was observed. Operation of the ADR requires some mechanism for thermally linking the salt pills to the heat sink, and then thermally isolating them. With the failed heat switch unable to perform this function, an alternate plan was devised which used carefully controlled amounts of exchange gas in the dewar's guard vacuum to facilitate heat exchange. The process was successfully demonstrated in November 2012, allowing the ADR to cool the detectors to $50 \mathrm{mK}$ for hold times in excess of $10 \mathrm{~h}$. This paper describes the exchange-gas-assisted recycling process, and the strategies used to avoid helium contamination of the detectors at low temperature.
\end{abstract}

Published by Elsevier Ltd.

\section{Introduction}

Astro-H [1], Japan's sixth X-ray astronomy mission, is currently the only major X-ray facility under development for use in space. Its four instruments will make observations across the X-ray spectrum $(0.3-600 \mathrm{keV})$ to investigate such topics as how matter behaves in extreme gravitational fields, how the largest structures in the Universe grew, and the spin rate of black holes. The most sensitive instrument is the Soft X-ray Spectrometer (SXS) [2], which will perform imaging spectroscopy with a resolution of better than $7 \mathrm{eV}$ in the $0.3-12 \mathrm{keV}$ band. This will be accomplished with a $6 \times 6$ array of microcalorimeters operating at $50 \mathrm{mK}$. The array is cooled by a 3-stage ADR [3] which thermally connects to both a $40 \mathrm{l}$ superfluid helium tank and a $4.5 \mathrm{~K}$ Joule-Thomson cryocooler. This configuration allows the ADR to operate using the

\footnotetext{
* Corresponding author.

E-mail address: peter.shirron@nasa.gov (P. Shirron).
}

liquid helium as a heat sink, and the JT cooler after the helium is depleted. The basis of the ADR's design was a hold time of $24 \mathrm{~h}$ at $50 \mathrm{mK}$, and a recycle time of $1-2 \mathrm{~h}$. These values are consistent with a system level observing efficiency of $>90 \%$.

The SXS dewar, including the cryocoolers, was designed and built by Sumitomo Heavy Industries, Inc., (SHI) Japan, and the detector array and ADR (collectively referred to as the Calorimeter Spectrometer Insert (CSI)), were designed and built by NASA/GSFC. The SXS instrument has significant heritage in the X-Ray Spectrometer (XRS) instrument on Astro-E [5] and Astro-E2 [6]. The major difference is that the solid neon dewar used for both XRS instruments has been replaced by a complex of Stirling and JT cryocoolers. This was done to achieve two objectives: redundancy in the cryogenic system, and longer mission life.

The extreme sensitivity of the SXS detectors, and the criticality of the SXS instrument to Astro-H mission science, led the project to propose building a full engineering model (EM) instrument that could be used to investigate possible coupling between the 
cryocoolers and the detector signals - from electromagnetic interference, exported vibration or other mechanisms. The EM program included extensive testing of the SXS instrument, both as an isolated subsystem and after integration onto the spacecraft. The latter provided an opportunity to investigate interference with the SXS detectors and other instruments, particularly the cryocooler used on the Soft X-ray Imager (SXI) instrument, and spacecraft components such as the reaction wheels. Corrective action could be taken prior to committing to the flight instrument design.

In late 2011, the EM CSI was assembled and subjected to qualification level consisting of pre-vibe performance testing, vibration testing, and post-vibe performance testing. At the time, there were no obvious changes in performance that could not be attributed to disassembly and re-assembly of the 2-stage ADR just prior to vibration testing. The EM CSI was delivered in March 2012 to SHI for integration into the EM dewar. The first cooldown of the EM instrument occurred in July 2012.

During the cooldown, one of the ADR's four gas-gap heat switches was observed to thermally decouple as the entire assembly slowly cooled through about $100 \mathrm{~K}$. The faulty heat switch (HS2 in Fig. 1) links the ADR's 2nd stage to the helium tank. The behavior of the heat switch suggested that its containment shell, consisting of an outer 2-ply layer of T300 carbon fiber composite and an inner 12 micron foil of Ti15333, had developed a significant permeation leak, and that all of the initial helium-3 charge had been lost and replaced by some fraction of an atmosphere of air. In any event, the ADR could not operate without the function of this heat switch, and the whole EM program was at risk.

Since the EM SXS hardware was also needed for spacecraft-level tests such as EMI/EMC compatibility testing and vibration qualification testing, and the schedule for these had little to no slack, disassembly of the dewar and repair or replacement of the failed heat switch was not practical. Therefore, any and all options for recovering some operation of the CSI were developed, including the use of helium exchange gas in the dewar's guard vacuum to thermally couple across the failed switch. While the latter is clearly a nonstandard operating mode for a high-performance dewar system, it represented - ultimately - the best and lowest risk approach to enabling the EM program to move forward. This paper describes the exchange gas-assisted ADR recycling process, and the results of its successful implementation.

\section{SXS cryogenic system}

The SXS cryogenic system [4], shown schematically in Fig. 1, consists of a small ( $40 \mathrm{l}$ ) helium tank, a $4.5 \mathrm{~K}$ JT cryocooler, two pairs of 2-stage Stirling cryocoolers, a 3-stage ADR, and the detector assembly. Two of the Stirling cryocoolers connect to an inner and an outer vapor cooled shield (IVCS and OVCS), which are also cooled by the helium boiloff gas. The other two Stirling cryocoolers act as pre-coolers for the JT system, which also cools a shield immediately surrounding the helium tank. The ADR and detector assembly are an integral unit that mounts to the top of the helium tank. The ADR is functionally divided into a 2-stage ADR (inside the $1.2 \mathrm{~K}$ shield) which cools the detectors using the liquid helium as a heat sink, and a 3rd stage ADR which pumps heat from the helium tank to the JT cooler.

With the failed heat switch, the salt pills in the 2-stage ADR do not have a direct, high-conductance path to the heat sink. Instead, they are thermally isolated from the helium tank except for the weak connection through the containment shell of heat switch HS2, sensor wiring, and Kevlar suspension components. This configuration is schematically shown in Fig. 2.

\subsection{Possible recovery options}

After discovery of the heat switch failure, three options for recovering some operation of the ADR were developed and evaluated.

Option A involved using the weak residual thermal coupling between the ADR and the helium tank to recycle the ADR. In this case, the ADR's salt pills would be magnetized to full field, warming them to as high as $20 \mathrm{~K}$, then they would eventually cool to a temperature at which the ADR could be demagnetized to $50 \mathrm{mK}$. The stages would have to cool to at least $5 \mathrm{~K}$, at which point the 2nd stage could precool the 1 st stage to about $2 \mathrm{~K}$, and then the 1st stage could reach $50 \mathrm{mK}$ with about $2 \mathrm{~h}$ of hold time - severely limited by the high boundary temperature of the 2nd stage and the high heat flow through HS1. Based on measurements of heat flow versus salt pill temperature, the time needed to reach $5 \mathrm{~K}$ was estimated to be 20 days.

In Option B, exchange gas would be admitted to the dewar's guard vacuum to enhance the thermal contact between the salt pills and components anchored to the helium tank - principally

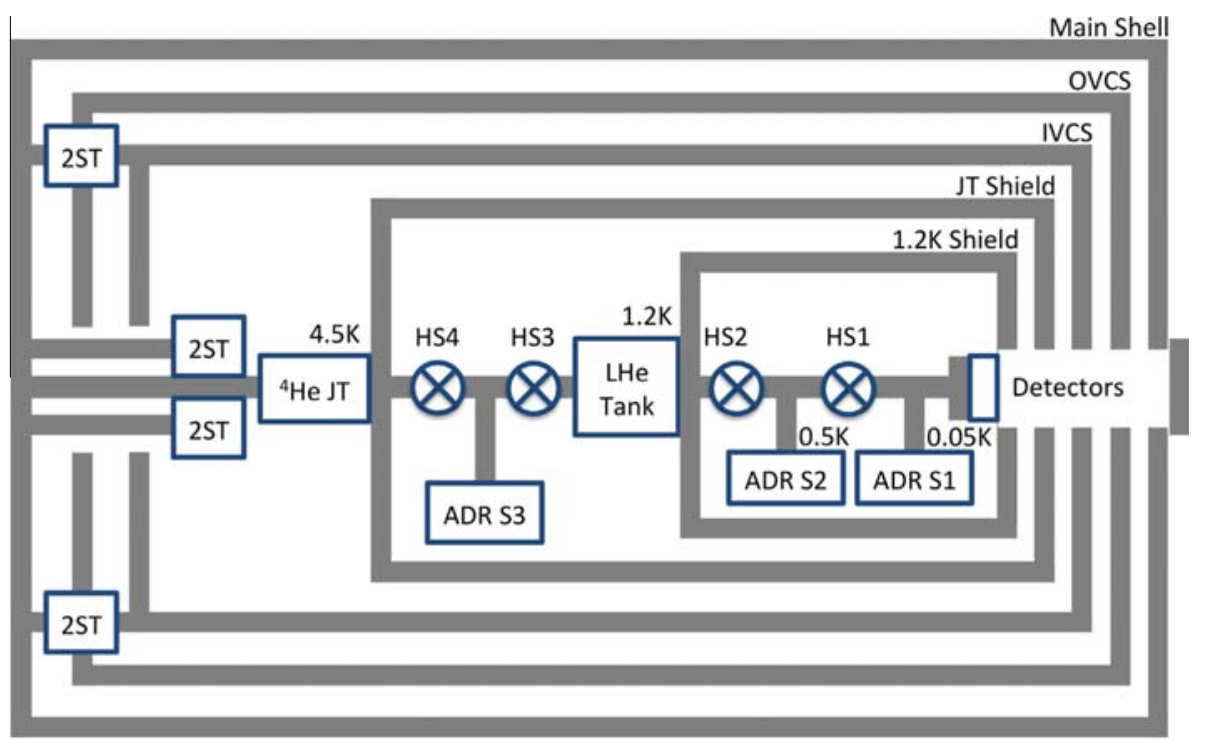

Fig. 1. Schematic representation of the SXS cryogenic system. 


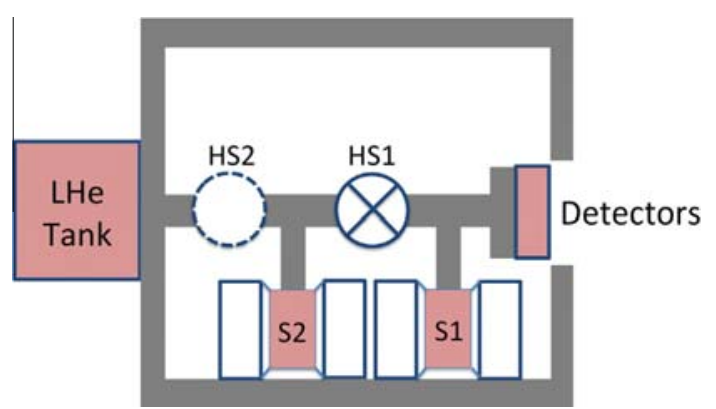

Fig. 2. Effective thermal schematic of the SXS helium tank and 2-stage ADR with the failed heat switch. Heat switch HS1, and the two salt pills (S1 and S2) and their magnets remained functional, but thermally decoupled from the helium tank.

their magnets. The ADR's salt pills would be magnetized and cooled to close to the helium bath temperature. After eliminating the exchange gas, the ADR could be progressively cooled as outlined in Option A. If the salt pills could be cooled to about $3 \mathrm{~K}$ at full magnetic field, the systems cooling capacity would be sufficient to allow it to reach $50 \mathrm{mK}$ with more than $10 \mathrm{~h}$ of hold time. Based on estimates of gas conductance in the $\sim 1 \times 10^{-3} \mathrm{~Pa}$ ra-oled to $3 \mathrm{~K}$ in less than $1 \mathrm{~h}$.

The concept of Option C was to backfill the dewar's guard vacuum with up to 1 atmosphere of helium- 4 and allow up to 1 week for helium to diffuse into the switch, possibly recovering full operation of the ADR. The main difficulty for this option was to bound the helium leak/permeation rate through the shell and assess whether the leakage would occur only near room temperature (characteristic of permeation) or at all temperatures (indicative of a leak). In either case, any helium injected into the switch would partially leak back out during the long period needed to clean up the guard vacuum. With a large enough leak (permeation or direct leak), the helium charge could dissipate before the ADR could be cooled down. If a direct leak were present, the heat switch might continue to degrade at low temperature at too fast a rate to provide useful function.

The relative merits of each option involved both objective measures of how well the ADR might perform, and subjective assessments of risk or compromise to the entire SXS system. Option A would yield the poorest ADR performance, but except for the need to run the ADR magnets continuously at full current for weeks, the operation imposed no apparent risk to the SXS hardware. Option B significantly increased the amount of "cold time" that could be achieved, but risked contaminating the guard vacuum in a way that could seriously degrade detector performance at $50 \mathrm{mK}$, and possibly compromise future measurements of the dewar's thermal performance. Option C could conceivably restore the ADR to full operation, but it might also yield nothing if the leak was too large. In either case, it was certain to heavily contaminate the guard vacuum.

The decision ultimately depended on the discovery of a very small helium leak in the dewar itself, which appeared after an extended time at superfluid conditions. At that point, the deliberate introduction of exchange gas for Options B and C no longer represented an irreversible compromise to the guard vacuum. Option $\mathrm{B}$ was chosen for implementation based on its higher likelihood of success and the lower likelihood of helium contamination once the detectors were cooled.

\section{ADR cycling using exchange gas}

\subsection{Gas thermal conduction}

Referring to Fig. 2, the introduction of helium exchange gas into the guard vacuum of a dewar containing an ADR will, at some level, thermally connect the salt pills to surrounding components. The strongest coupling will be to the magnets and magnetic shields which surround the salt pills, and from which they are separated by a uniformly small gap. For heat flow at low temperature in the molecular regime, we use an expression by Kumagai et al. [7], although similar formulations may be found in White and Meeson [8]. Heat flow per unit area between surfaces at $T_{1}$ and $T_{2}$ is

$\frac{\dot{Q}}{A}=\frac{1}{2}\left(\frac{\gamma+1}{\gamma-1}\right) \sqrt{\frac{k_{B}}{2 \pi m}} \frac{T_{1}-T_{2}}{\left(\sqrt{T_{1}}+\sqrt{T_{2}}\right) / 2} P_{R T}$

where $\gamma$ is the ratio of specific heats at constant pressure and volume, $m$ is the mass of atoms/molecules of the gas, and $P_{R T}$ is the pressure in the system, measured at room temperature. In SI units, where pressure is given in Pa and temperature in $\mathrm{K}$, the expression, for helium gas, reduces to

$\frac{\dot{Q}}{A}=\left(74.0 \mathrm{~W} / \mathrm{m}^{2}\right) \frac{T_{1}-T_{2}}{\sqrt{T_{1}}+\sqrt{T_{2}}} P_{R T}$

This expression specifies the maximum possible heat flow between the surfaces, since transfer of heat requires the gas to stick to each surface it strikes. In practice, this expression is found to closely approximate heat transfer between the salt pills and magnets, possibly due to the small gap which enhances the frequency of bounces if a helium atom is not adsorbed.

For example, for a pressure of $10^{-3} \mathrm{~Pa}$, and a salt pill at $5 \mathrm{~K}$ inside a magnet at $3 \mathrm{~K}$, the heat flow per unit area would be $37 \mathrm{~mW} / \mathrm{m}^{2}$. The 1 st stage salt pill has a surface area of $0.027 \mathrm{~m}^{2}$, and the 2nd stage salt pill $0.015 \mathrm{~m}^{2}$. The combined heat flow from both salt pills can exceed $1.5 \mathrm{~mW}$. At this rate, the several Joules of heat that must be rejected from the ADR can be transferred in less than $1 \mathrm{~h}$.

However, the exchange gas causes heat flow between other components of the system, particularly the shields connected to the cryocoolers. At too high a pressure, the heat flow will overwhelm their cooling power. Thus a balance of adequate heat exchange for the salt pills and acceptable heat loads on the cryocoolers and helium tank demanded that the pressure be kept in the $1-2 \times 10^{-3}$ Pa range.

In fact, because the JT cooler shield (at $4.5 \mathrm{~K}$ and $>1 \mathrm{~m}^{2}$ area) directly faces a shield at $\sim 26 \mathrm{~K}$, even for pressures as low as $10^{-4} \mathrm{~Pa}$, its heat load would exceed the approximately $20 \mathrm{~mW}$ of excess cooling capacity. For this reason, Option B was not considered feasible without liquid helium in the tank to stabilize the system.

\subsection{Helium contamination}

While the exchange gas is clearly vital to charging the ADR, it must be eliminated before cooling to sub-kelvin temperatures. Any residual gas in the guard vacuum would condense onto the ADR and detectors as they cooled below the tank temperature. Surface coverages of even one monolayer would severely degrade the resolution of the detectors through the added heat capacity.

Pumping on the guard vacuum is eventually effective, but even in the most open systems, several days would be required to reach sufficiently low pressure. The tightly layered shields and MLI in the SXS dewar necessitated a different approach: the use of the helium tank as a gettering surface for the exchange gas. The vapor pressure above the adsorbed film on the cold surfaces is an extremely strong function of temperature, and very modest cooling of the helium tank will reduce the pressure to levels that are undetectable on either an external pressure gauge or a helium leak detector.

This can be seen by casting the chemical potential, $\mu$, of the adsorbed film in the Frenkel-Halsey-Hill [9] formulation as 
$P=P_{\text {svp }} e^{-\mu / k_{B} T}$

where $P$ is the pressure above the film and $P_{s v p}$ is the saturated vapor pressure at temperature $T$. Both terms in Eq. (3) have an exponential dependence on $T$, resulting in $P$ potentially having an extremely strong dependence on temperature. The dependence can be far less dramatic if the film thickness, $d$, increases significantly as helium gas is adsorbed, given that $\mu-d^{-3}$ [10]. However, in the regime required for this operation, vapor pressures are necessarily small, on the order of $10^{-8}$ atm. Even with a very conservative estimate of the effective (i.e. cold) volume of the guard vacuum, the amount of He in the gas phase is less than $1 \%$ of the amount adsorbed onto the cold surfaces, and therefore the film thickness does not increase appreciably as gas is adsorbed.

The effect on vapor pressure of reducing tank temperature can be seen in Fig. 4, in which an external pressure gauge was used to monitor guard vacuum pressure after dosing approximately 1 standard $\mathrm{cm}^{3}$ of He gas into the engineering model dewar. Cooling the tank by only $10-15 \%$ produced an order of magnitude change in vapor pressure, and when cooling below $2 \mathrm{~K}$, the helium signal from the dewar was undetectable.

The strong dependence of vapor pressure on temperature also means that the amount of gas absorbed onto the tank when the exchange gas is present is a strong function of temperature. Consequently, there was concern that if the temperature chosen for transferring heat from the ADR was too low, the thicker film would not allow the vapor pressure to be reduced low enough to prevent contamination of the detectors at $50-60 \mathrm{mK}$. On the other hand, if the transfer temperature is too high, the cooling capacity achieved might not be enough to reach $50 \mathrm{mK}$ with acceptable hold time. As a compromise, cycling was performed at $3 \mathrm{~K}$, and the tank was subsequently pumped to less than $1.3 \mathrm{~K}$.

After cooling the tank, a very thin film of helium would almost certainly still be adsorbed on the detectors, and potentially enough to degrade their performance through the added heat capacity. Consequently, a "degassing" operation was included in which the detectors were warmed above $6 \mathrm{~K}$ for $10 \mathrm{~min}$ before demagnetization to low temperature. In the high vacuum environment produced by the pumped helium tank, the helium coverage on the detectors at $6 \mathrm{~K}$ was calculated to be negligible - a fact that was confirmed during the first ADR cycle.

\subsection{Complete ADR cycling process}

The ADR cycling process itself consisted of only a few basic steps. The starting point was to pump the helium tank to $3 \mathrm{~K}$, as measured at the top where the ADR is located. The salt pills were typically about $4-5 \mathrm{~K}$ at the end of the dewar cooldown. In cases where they were warmer, they began cooling once the dosing of exchange gas began, and cooled below $5 \mathrm{~K}$ in less than $30 \mathrm{~min}$.

Exchange gas was dosed into the guard vacuum until the pressure on an external gauge read steadily at the desired value, as evidenced by steady cooling of the salt pills toward $3 \mathrm{~K}$. For the majority of cycles performed, the target value was $2 \times 10^{-3} \mathrm{~Pa}$. One of the complicating factors was that the exchange gas caused the top of the tank, which is not in direct contact with liquid, to warm relative to the bottom. Over time, the gradient tended to increase, causing exchange gas to adsorb onto the tank bottom and reduce the heat flow. Careful control of the pumping rate was needed to keep the tank top at $3 \mathrm{~K}$, and a mass gauge heater at the bottom of the tank was powered in order to minimize the gradient across the liquid.

The controllers for the two ADR stages were set to control the salt pill temperatures at $5 \mathrm{~K}$. At this point, turning on HS1 is advantageous since far more heat is generated by the magnetization of the 2 nd stage, and the 1 st stage has the larger surface area and heat flow capability. Magnetization continues until the magnets reach full current, and the salt pills cool close to $3 \mathrm{~K}$. The helium tank is then pumped to $<1.3 \mathrm{~K}$, and the exchange gas is completely adsorbed onto the tank surface. Fig. 3 shows the pressure read out of the external gauge during this process.

At this point, the entropy (or cooling) capacity of the ADR is fixed. That is, the maximum hold time for the detectors at low $T$ is now determined. In subsequent steps, the cooling capacity is moved between the stages in order to accomplish two objectives: to degas any residual helium from the detectors, and to prepare the 1 st stage for final demagnetization cooling. Cooling capacity is transferred between stages by using one of the stages to the other down, using each stage's temperature controller to maintain a moderate temperature difference (to maintain high transfer efficiency) across HS1.

To perform the degassing operation, (1) the 1st stage transfers all of its cooling capacity to the 2nd stage through HS1, (2) HS1 is opened, (3) the 1st stage is magnetized to full current, which raises its temperature from $\sim 3 \mathrm{~K}$ to $>6 \mathrm{~K}$, for $10 \mathrm{~min}$, and (4) the stage is demagnetized to zero current, back to $\sim 3 \mathrm{~K}$.

At the end of the degassing process, the 2nd stage has essentially all of the stored cooling capacity of the 2-stage ADR (charged to $3 \mathrm{~T}$ at typically $2.5 \mathrm{~K}$ ). The majority of this cooling capacity is transferred back to the 1 st stage to set up for its demagnetization to $50 \mathrm{mK}$. Heat is flowed between the stages, charging the 1st stage to $2 \mathrm{~A} / 2 \mathrm{~T}$ and cooling it to below $2 \mathrm{~K}$. Some capacity is retained in the 2nd stage so that it can demagnetize to a lower temperature during the hold and significantly reduce the parasitic heat flow through HS1.

Once the transfer is complete, both stages are demagnetized to their hold temperatures: $0.9-1.0 \mathrm{~K}$ for the 2 nd stage and $50-60 \mathrm{mK}$ for the 1 st stage.

The complete recycling process, and the subsequent hold period are shown in Figs. 5 and 6. The time required for the full recycling process exceeded a normal work shift, so it was divided into three periods. The initial transfer of heat from the ADR stages to the helium tank was performed during one work shift. After pumping the helium to $<2 \mathrm{~K}$, the ADR was left idle for up to $16 \mathrm{~h}$, during which time some additional cooling occurred through the Kevlar suspension and heat switch structures. During the subsequent work shift, the detectors were degassed and the ADR was prepared for cooldown. Combined, the recycling process took up to $30 \mathrm{~h}$ to complete - as opposed to $1 \mathrm{~h}$ for a normally operating ADR.

Since each cycle achieved a hold time of only 9-16 h at 50$60 \mathrm{mK}$ (compared to 42-74 h demonstrated before the failure of the heat switch), test sequences were carefully scheduled to make full use of the available cooling capacity. Fortunately, breaks in testing could be introduced by temporarily warming the ADR and detectors to $\sim 0.5 \mathrm{~K}$, where the usage of entropy capacity was negligible.

When the 1st stage ADR ran out of cooling capacity, the next cycle could be started by warming the helium tank back to $3 \mathrm{~K}$. As the tank warms above about $2.4 \mathrm{~K}$ (as seen in Fig. 3), the adsorbed exchange gas is released, thermally connecting the salt pills and helium tank.

After a few cycles were performed, some improvements were made to make the process more time-efficient. Since the transfer rate depends on surface area, more heat is rejected from Stage 1 than Stage 2. As the salt pills cooled, a faster time-average transfer rate could be achieved by periodically transferring the cooling capacity in Stage 1 to Stage 2 by flowing heat from Stage 2 to Stage 1 through HS1, then opening HS1 and magnetizing Stage 1 to a higher temperature. The process allows both salt pills to be cooled slightly below the tank temperature in about $2 \mathrm{~h}$, as opposed to cooling to within about $0.1 \mathrm{~K}$ of the tank in $4-5 \mathrm{~h}$. The oscillation 

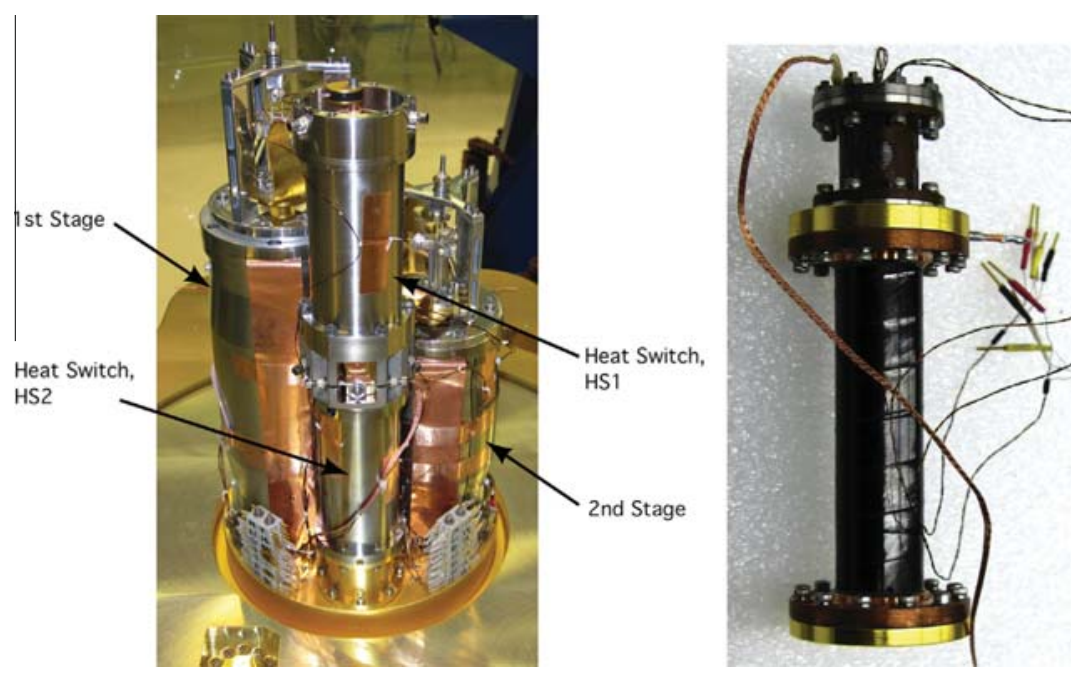

Fig. 3. The 2-stage ADR (left) and HS2 (right) with its T300 carbon fiber composite shell. The shell is approximately $8 \mathrm{~cm}$ long and $1.8 \mathrm{~cm}$ diameter.

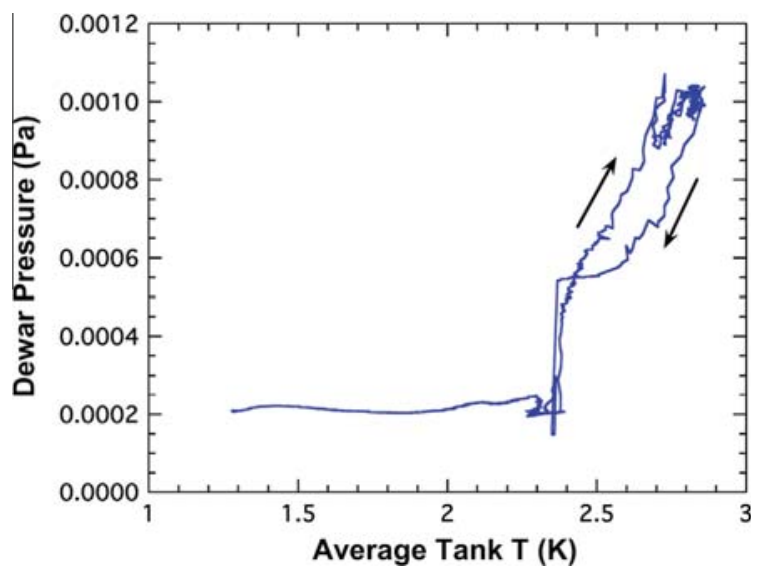

Fig. 4. Vapor pressure in the guard vacuum, measured on an external gauge, as a function of the average tank temperature, for both increasing and decreasing temperature. The background reading for the gauge was $2 \times 10^{-4} \mathrm{~Pa}$.

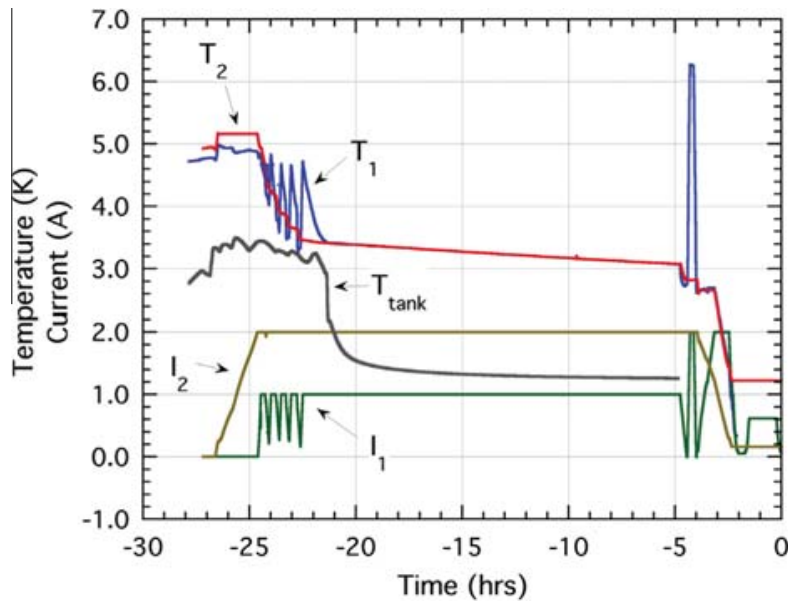

Fig. 5. Temperature and current during the recycling portion of one gas-assisted ADR cycle. Time is zero at the beginning of the hold time. Subscripts for $T$ and $I$ denote the respective stage; $T_{\text {tank }}$ is the temperature of the tank where the ADR is located.

in Stage 1's current and temperature before the helium tank is cooled below $3 \mathrm{~K}$ reflects this periodic transfer.

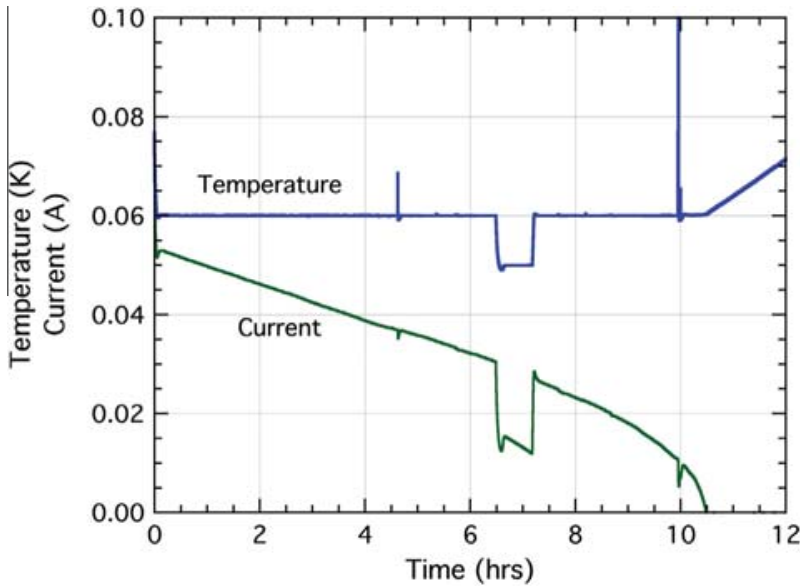

Fig. 6. Temperature and current in the 1 st stage during a hold time. The temperature setpoint was either $50 \mathrm{mK}$ or $60 \mathrm{mK}$.

\section{Results}

Recycling the SXS EM ADR using exchange gas was successfully demonstrated on the first attempt, in November 2012. Since then 13 cycles have been completed, each time providing 8-13 h of test time at $50-60 \mathrm{mK}$. The cycles have been conducted in groups of 23 , each time requiring about 1 week of schedule. The primary goal was to identify coupling, if any, between the cryocoolers and detectors on the SXS instrument, and secondarily to identify coupling between other instruments and spacecraft components. Consequently, ADR cycles were conducted with the SXS instrument at several different levels of assembly, from the dewar level up to the full spacecraft level with all Astro-H instruments present.

On the first cycle, after cooling to $50 \mathrm{mK}$, coupling between the cryocoolers and the SXS detectors was clearly observed. It appeared as both excess noise in the measurement of X-ray energies - resulting in a degraded resolution of $20-30 \mathrm{eV}$ (for $5.9 \mathrm{keV}$ Fe55 X-rays) instead of the expected $4-7 \mathrm{eV}$ - and as thermal noise in both the SXS detector and ADR stage temperatures. The latter led to speculation that the coupling was primarily mechanical heating of components at $50 \mathrm{mK}$. What made the mechanical heating problematic was that it exhibited a randomness over time scales of seconds to tens of seconds that could not be damped out by the ADR's temperature controller. 


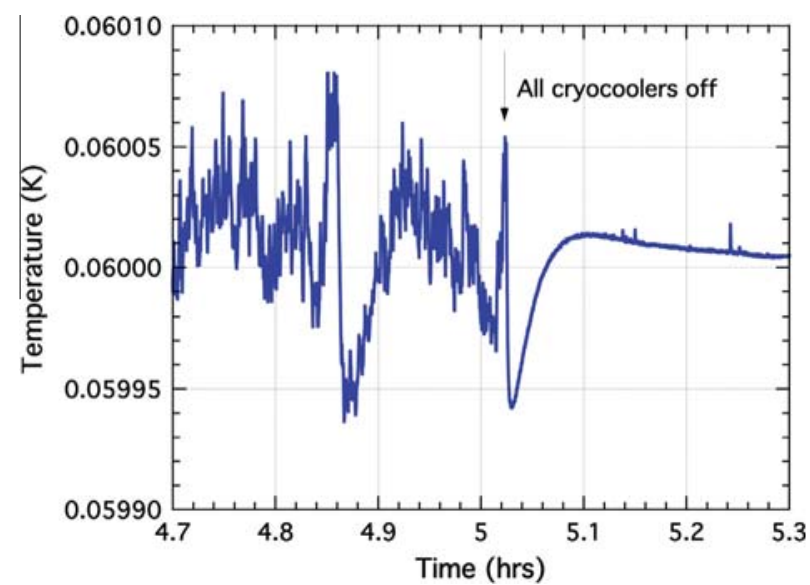

Fig. 7. Temperature noise is dramatically reduced as the cryocoolers were progressively turned off.

When all cryocoolers were turned off, as seen in Fig. 7, the temperature stability immediately improved, nearly to the levels demonstrated in subsystem tests at Goddard Space Flight Center before delivery to Japan. An effort is now underway to develop mechanical isolation structures for the cryocooler compressors to reduce exported vibration, and to minimize the overlap of sensitive frequencies for the detector system with peaks in the cryocooler vibration spectra. These will be partially implemented on the engineering model hardware, and, if successful, fully implemented in the flight design.

\section{Summary}

The failure of one of the four heat switches in the engineering model 3-stage ADR built for the Astro-H SXS instrument led to an exhaustive search for recovery options and evaluation of each one's potential to damage or compromise the SXS hardware. Ultimately the option chosen, both for its higher potential for successfully cooling the ADR to $50-60 \mathrm{mK}$ and the ability to obtain significantly more test time at low temperature, was to use helium-4 exchange gas to replace the function of the failed switch. While it provides beneficial heat exchange between ADR components and the helium tank, the exchange gas also generates unwanted heat flows within the remainder of the cryogenic system. Fortunately, pressures in the $1-2 \times 10^{-3} \mathrm{~Pa}$ range provide adequate thermal conduction within the ADR assembly to allow recycling, but not so much conduction among warmer components that the cryocoolers or helium tank are overwhelmed.

The process relied critically on being able to eliminate the exchange gas after charging the ADR stages, and this was accomplished by cooling the helium tank and adsorbing the gas onto the tank surface. The residual pressure was low enough that, after a degassing operation at $6 \mathrm{~K}$, detectors sensitivity at $50-60 \mathrm{mK}$ was indistinguishable from that observed in performance tests when the ADR was functioning normally. Even after extended operation at low temperature, the detectors never showed any sign of helium loading, which would have been evident if even a very small fraction of a monolayer of helium had condensed on their surface.

The successful implementation of this ADR recycling technique allowed the main goals of the Astro-H/SXS engineering model program to be met, including probing coupling between the SXS cryocoolers and detectors. As a result, significant compatibility issues were identified early, and mitigation strategies could be demonstrated before finalizing the design of the flight instrument.

\section{References}

[1] Takahashi T et al. The ASTRO-H mission. In: Proc SPIE 7732, space telescopes and instrumentation 2010: ultraviolet to gamma ray, 2010. p. 77320Z77320Z-18.

[2] Mitsuda K et al. The high-resolution X-ray microcalorimeter spectrometer system for the SXS on ASTRO-H. In: Proc SPIE 7732, space telescopes and instrumentation 2010: ultraviolet to gamma ray, 2010. p. 773211-773211-10.

[3] Shirron P, Kimball M, James B, Wegel D, Martinez R, Faulkner R, et al. Design and predicted performance of the 3-stage ADR for the Soft-X-ray Spectrometer instrument on Astro-H. Cryogenics 2012;52(4-6):165-71.

[4] Fujimoto R et al. Cooling system for the Soft X-ray Spectrometer (SXS) onboard ASTRO-H. In: Proc SPIE 7732, space telescopes and instrumentation 2010: ultraviolet to gamma ray, 2010.

[5] Breon SR, Branch HD, Blount GJ, Jackson ML, Boyle RF, Tuttle JG. Design of the XRS helium insert. Adv Cryog Eng 1996;41:1129-34.

[6] Kelley R et al. The Suzaku high resolution X-ray spectrometer. Publ Astron Soc Jpn 2007;59:S77-S112.

[7] Kumagai H, Tominaga G, Tuzi Y, Horikoshi G. Vacuum science and engineering. Tokyo: Shokabo; 1970. p. 72.

[9] White GK, Meeson PJ. Experimental techniques in low-temperature physics. 4th ed. Oxford: Oxford Science Publications; 2002. p. 82.

[9] Frenkel J. Kinetic theory of liquids. Oxford: Oxford University Press; 1946. p. 332.

[10] Sabisky ES, Anderson CH. Phys Rev A 1973;7:790. 